\title{
An Internet of Things (IoT) based Monitoring System for Oil-immersed Transformers
}

\author{
O. YAMAN and Y. BICEN
}

\begin{abstract}
While the electricity power industry in the world continues to grow, it also becomes more traceable and smart with the developing technology. Naturally, the integration of these technologies into the electrical power systems brings with an additional cost. Most of the time producers and consumers struggle under the pressure of these additional costs and try new products that will reduce the cost. In this sense, competitive products in the market must be advantageous in terms of cost. In this study, an original IoT based transformer monitoring system has been developed by using open source software, modular and low cost components as an alternative to studies in literature. Arduino Mega 2560 microcontroller has been used as the main component in the system. The system can acquire data from a wide variety of sensors using internal and external transducers. And this data can be saved to the cloud system synchronously with the memory card on the monitoring system. The developed monitoring system has been utilized free and reliable platforms such as the ThingSpeak web interface and PushingBox Notification application. The all test process of the developed monitoring system has been carried out in the laboratory environment on a porotype transformer.
\end{abstract}

Index Terms - Internet of Things, IOT, Lifetime, Monitoring, Transformer

\section{INTRODUCTION}

$\mathrm{T}_{\mathrm{l} e \mathrm{~s}}^{\mathrm{H}}$ HE CONTINUOUS increase in the world population leads to an increase in energy demand. That means an additional capacity increase for those existing in electrical power systems. One of the most important parts of the energy transmission and distribution ring is oil filled transformer and affected by these capacity increase [1-3]. For this reason, it is very important to monitor the load conditions and operational performance of the transformers [4,5]. Also, demand for smart grid applications is increasing all over the world with the technological development [6-8]. Since the monitoring technologies are a sector with high $R \& D$ investments, the costs of the technologies produced are quite high [9-12]. In addition, many different companies develop different

YUNUS BICEN, is with Department of Electrical-Electronics Engineering, University of Duzce, Duzce, Turkey,(e-mail: yunusbicen@duzce.edu.tr).

(iD) https://orcid.org/ 0000-0001-8712-2286

OKAN YAMAN, is with Institute of Science and Technology, University of Duzce, Duzce, Turkey, (e-mail: yaman41okan@gmail.com).

iD https://orcid.org/ 0000-0002-4074-4050

Manuscript received February 9, 2019; accepted June 11, 2019. DOI: $10.17694 /$ bajece. 524921 monitoring systems in their own area of expertise [13-14]. When several monitoring systems are used together, costs can be very high. Although low-cost applications are limited in the literature, they have become popular. These applications are generally focused on monitoring technologies. It is very limited in terms of intervention, diagnostics or recognition. In a similar application, the current, voltage parameters for the distribution transformers were monitored by the ThingSpeak web interface [15]. In another study, the data were monitored using the low cost ESP8266 wireless module and Arduino Uno microcontroller [16]

In this study, it is aimed to develop a low cost and open source monitoring system that can be an alternative to high cost monitoring systems in the literature. The main advantage of using the open source software of the monitoring system is that it allows the user to modify and improve the system software. Developed system is based on Internet of Things (IoT) and is intended to perform the tasks defined in the electronic card. The open source Arduino Mega 2560 microcontroller developed by Atmel has been used in the transformer monitoring system. The Arduino development environment (IDE) is based on "Processing" and the Arduino programming language is based on "Wiring". The data received from the sensors on the transformer can be stored in the monitoring system and/or can be sent to the cloud system. Also, this data can be monitored via user-defined computers, tablets and phones. The monitoring system gives warning and informs the operator when the safe operating limit values are exceeded. These limit values, which are defined as default in accordance with the standard, can be easily updated on software in accordance with the information and approval of the operator. There are input modules for sensors on the board as well as output modules that can perform different tasks.

In this paper, the components of transformer monitoring system have been defined at section 2; the ThingSpeak web interface integration has been introduced used for the monitoring system at section 3, the PushingBox Notification application has been described at section 4, The details of the aging and lifecycle monitoring have been presented at section 5 , and the final section contains the conclusions and recommendations, respectively.

\section{TRANSFORMER MONITORING SYSTEM DESIGN}

While designing the monitoring system, it has been aimed to be fulfilling five key functions. Firstly, it should be possible to get the data from the sensors with different input parameters 
and to perform instant monitoring. Secondly, it should be possible to save this data both on its own system and on the cloud system. Thirdly, it should be ensured that analytical procedures can be performed on the system and the limit values related to the measured quantities are identifiable. Fourthly, in case of exceeding the limit values specified by the operator, he / she must fulfil the warning / information tasks and activate the pre-defined output relays. Finally, it has been expected to show the aging condition and the remaining service life estimation in accordance with the IEEE standard.

In this context, monitoring system components that can perform the specified functions have been determined and a general concept scheme has been created as shown in Fig. 1. Then, the flow chart of the monitoring system has been composed as shown in Fig. 2.
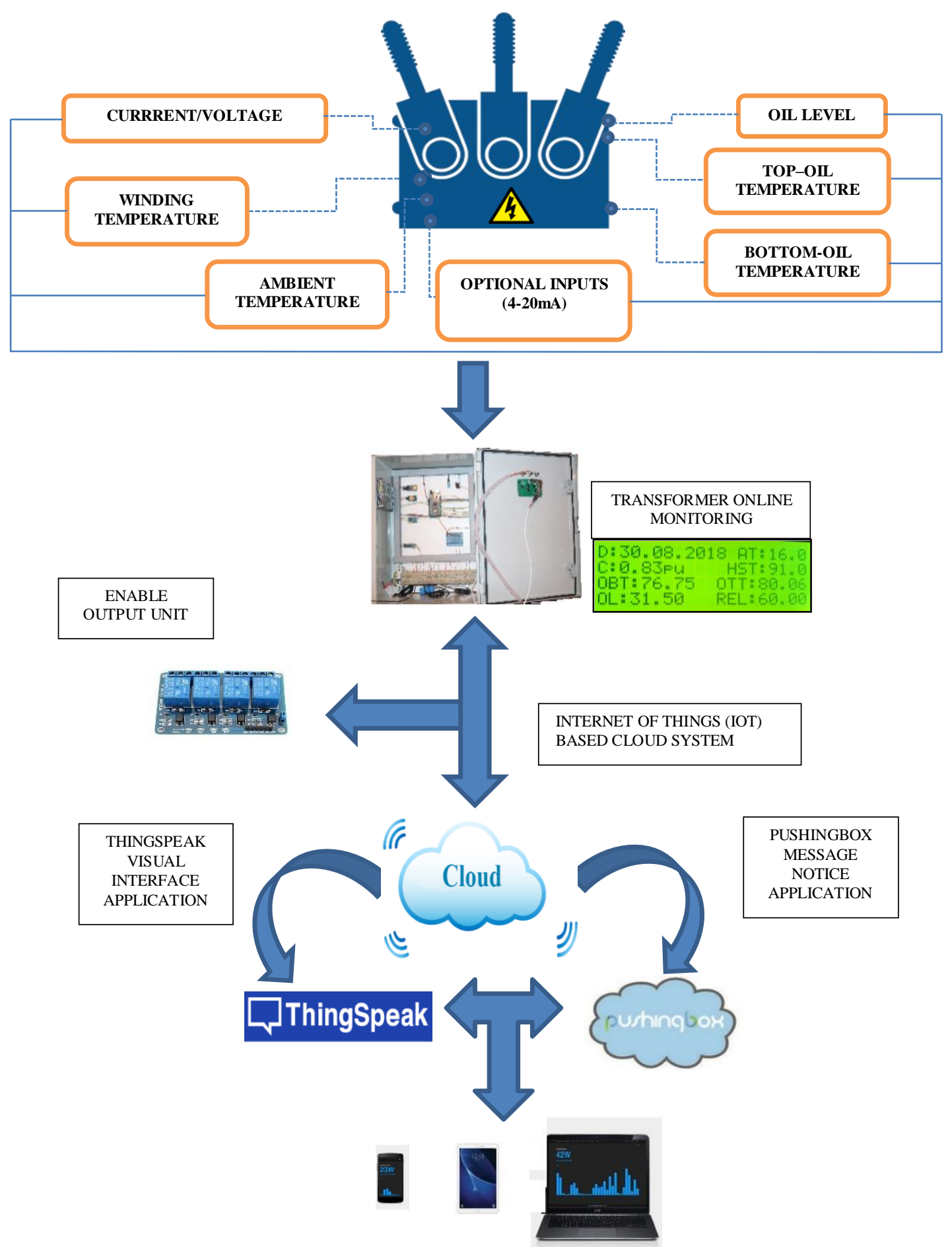

PHONE / TABLET / PC MONITORING

Fig. 1. Block diagram of transformer monitoring system 


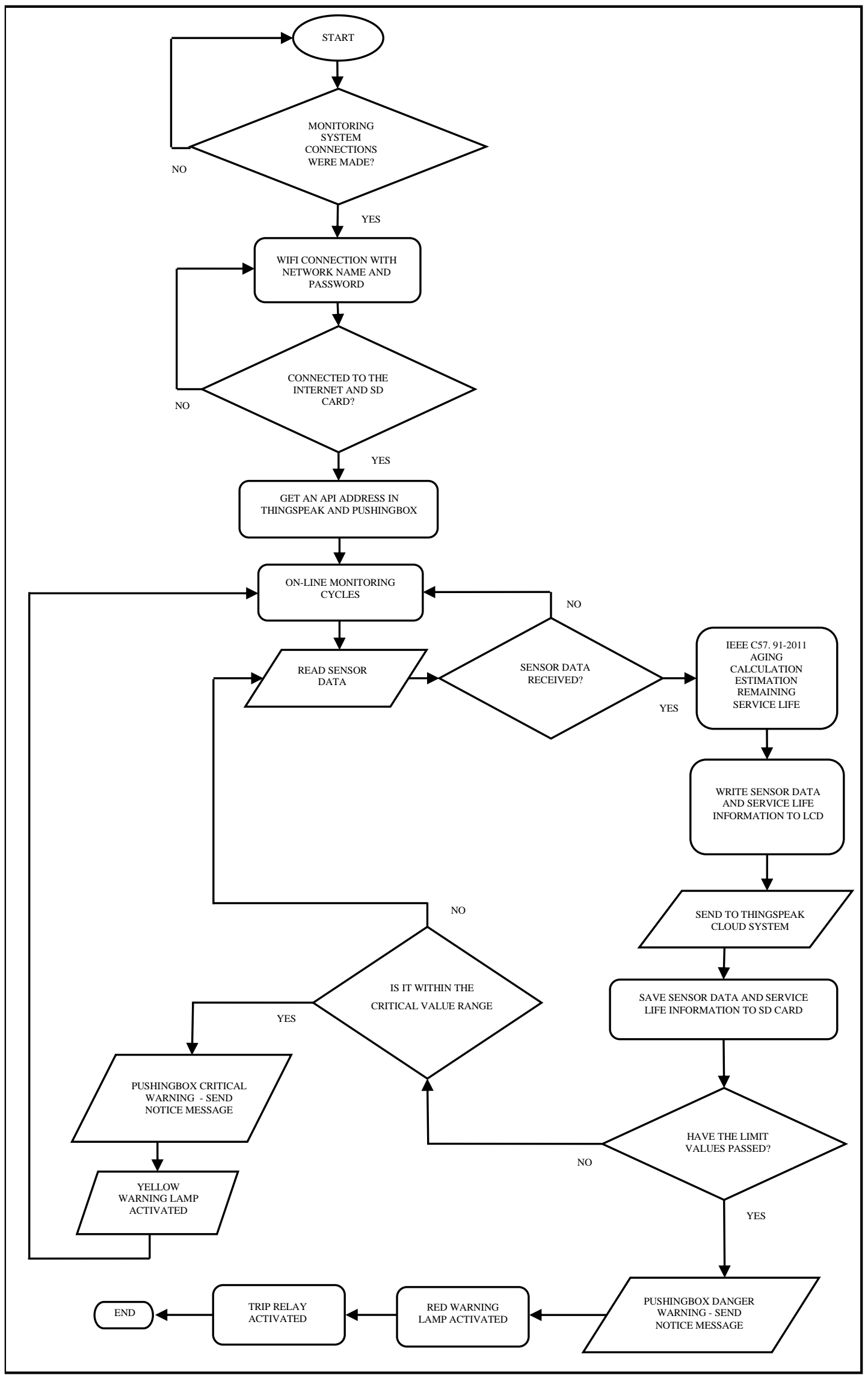

Fig. 2. Transformer monitoring system flow-chart 
The analogue signals accrued from different sensors installed on the transformer have been scaled between $0-5 \mathrm{~V}$ using transducer modules. Many parameters with standard sensor signals such as temperature (ambient, oil and hot-spot), current / voltage, insulation fluid level and so on can be defined as input. In this study, the temperature values at different points have been obtained by different sensors. The best measurement for hot-spot is done with fiber-optic sensors $[18,19]$. However, it is still a disadvantaged option considering the cost-benefit relationship [20]. Therefore, $\mathrm{K}$ type thermocouple with a precision of 0.25 degrees Celsius and rapid response time has been preferred for acquiring the temperature data from hot-spot. The digital temperature sensor DS18B20, manufactured by Dallas, has been used for oil and oil. This sensor is a very good option for its compatibility with the microcontroller, its ease of use and its resolution of up to
12 bits. The DHT11 digital sensor is used to measure the ambient temperature. This sensor which is capable of measuring with a resolution of 8 bits by $2 \%$ error margin between 0-50 degrees Celsius has been preferred because of its low cost and it can also receive the ambient humidity data optionally. A magnetic float level sensor has been used to measure the oil level. It has been made of heat-resistant stainless steel that producing an analogue output of $0-5 \mathrm{~V}$. As the current sensor, the non-invasive AC SCT-013 model, which was developed by YHDC, has been preferred. This sensor output is between $0-50 \mathrm{~mA}$ and can be used to measure $\mathrm{AC}$ currents up to a maximum of $100 \mathrm{~A}$. In addition to being compatible with microcontroller, it provides ease of use thanks to its clamp structure. A simple RC filter has been added to the sensor output in order to provide accurate values for the measurement results against external interference [21].

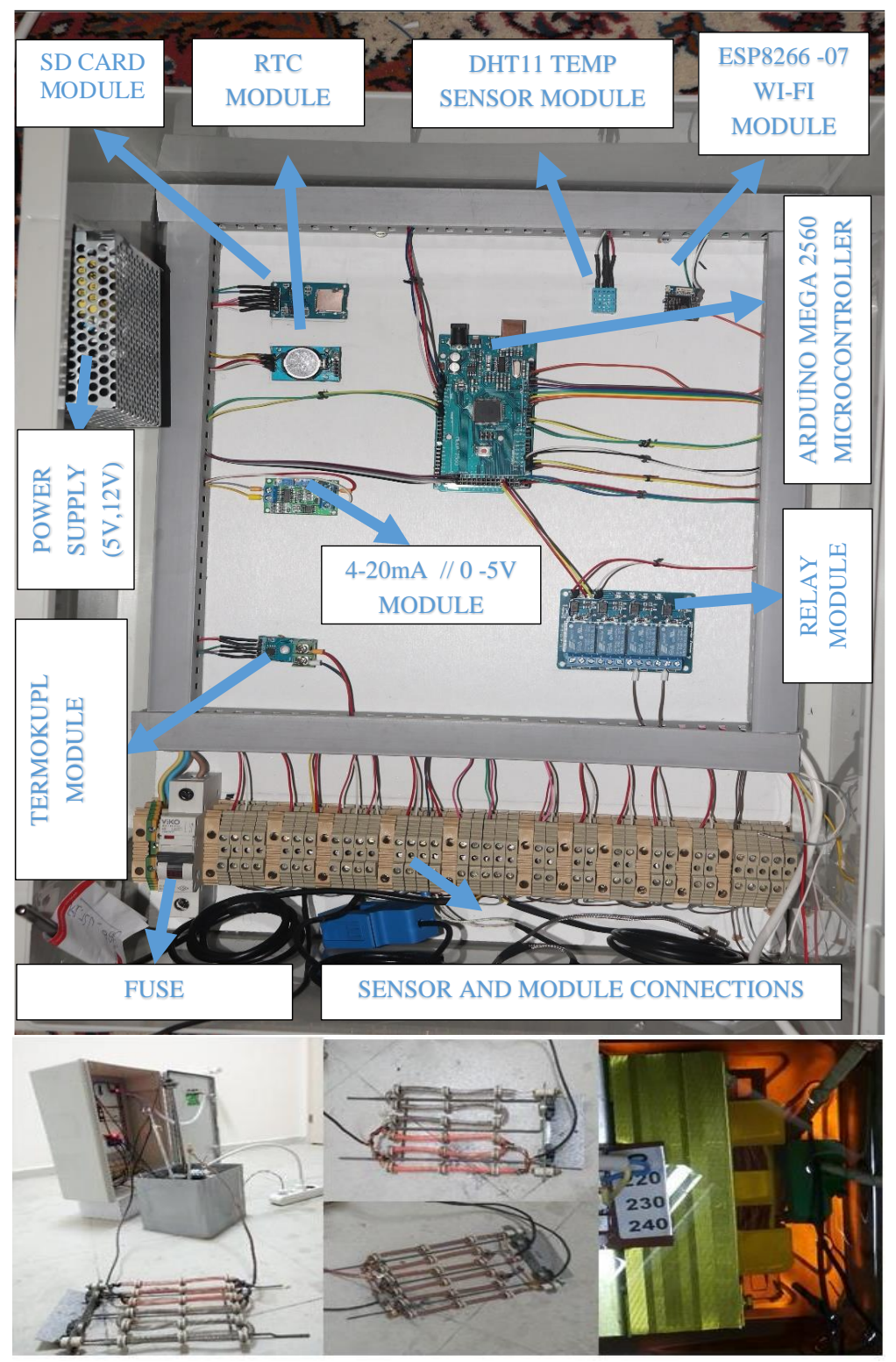

Fig. 3. Transformer monitoring system and test equipment 
Different parameters which cannot be taken as data in this study can be included with expansion modules in the following periods. In order to integrate sensors with a $4-20 \mathrm{~mA}$ output to the monitoring system, an analogue transducer module from 0 to $5 \mathrm{~V}$ has been used.

The mini SD card module has been used with the RTCDS3231 module in order to store the data received from the transformer with date and time information. The RTC-DS3231 module supports the I2C communication protocol. The ESP8266-07 series has been chosen as a Wi-Fi module for wireless transmission of sensor data to the cloud. This module has low power consumption and low cost. It also has a built-in antenna and supports the TCP / IP protocol. With this module that supports the Internet of Things (IOT), the data received from the sensors have been easily transferred to the ThingSpeak web interface. Also, the measured parameters of the transformer can be monitored on the panel of the system with the 20x4 LCD display. Yellow and red led, which are activated in early warning and emergency situations, have been used on the panel. In similar emergency situations, the output relays on the panel are also activated in order to perform the functions previously defined by the operator according to the limit values. In the monitoring system, $50 \mathrm{~W}$ switching power supply with $5 \mathrm{~V}$ and $12 \mathrm{~V}$ output and a fuse for system safety have been used. The inside of the completed panel of the monitoring system has been shown in the Fig. 3.

\section{The ThingSpeak Web InTERface}

In recent two decades, with the rapid development of internet technology, it has become possible to design different monitoring and control systems. One of these technologies is the Internet of Things (IoT). This structure is based on the idea of collecting, analyzing, making decisions and managing the data of the environment in which the objects are located on a common platform [22,23]. ThingSpeak is an open IOT platform using for real-time monitoring of the data and the data accrued from the sensors can be monitored by means of a user-defined IP address and ThingSpeak API key [24]. Any other user can see or download the data by using ThingSpeak at any location, if the system operator uses the public IP address. The user can also add location information.

In this study, 2 API addresses have been defined in ThingSpeak interface as shown in Fig. 4. These have been used for transformer online monitoring system Fig. 5. The transformer online monitoring system interface shows realtime winding (hot-spot) temperature, oil bottom, top-oil and ambient temperatures, oil level and current data from the sensors. It is observed that the ambient temperature is constant in the test environment. When the transformer load is increased in a controlled manner, it is seen that the winding temperature increases with the current. Increased winding temperature increases the aging factor. The aging condition and the remaining service life estimation have been calculated by reference to the hot spot temperature. At the oil level, there was no change during the test. The data accrued from the sensors and then calculated parameter changes have been shown in the ThingSpeak Graphical Interface. Fig. 6 shows that the same data can also be accessed via the mobile device. In this way, the system is constantly under the control of the operator.

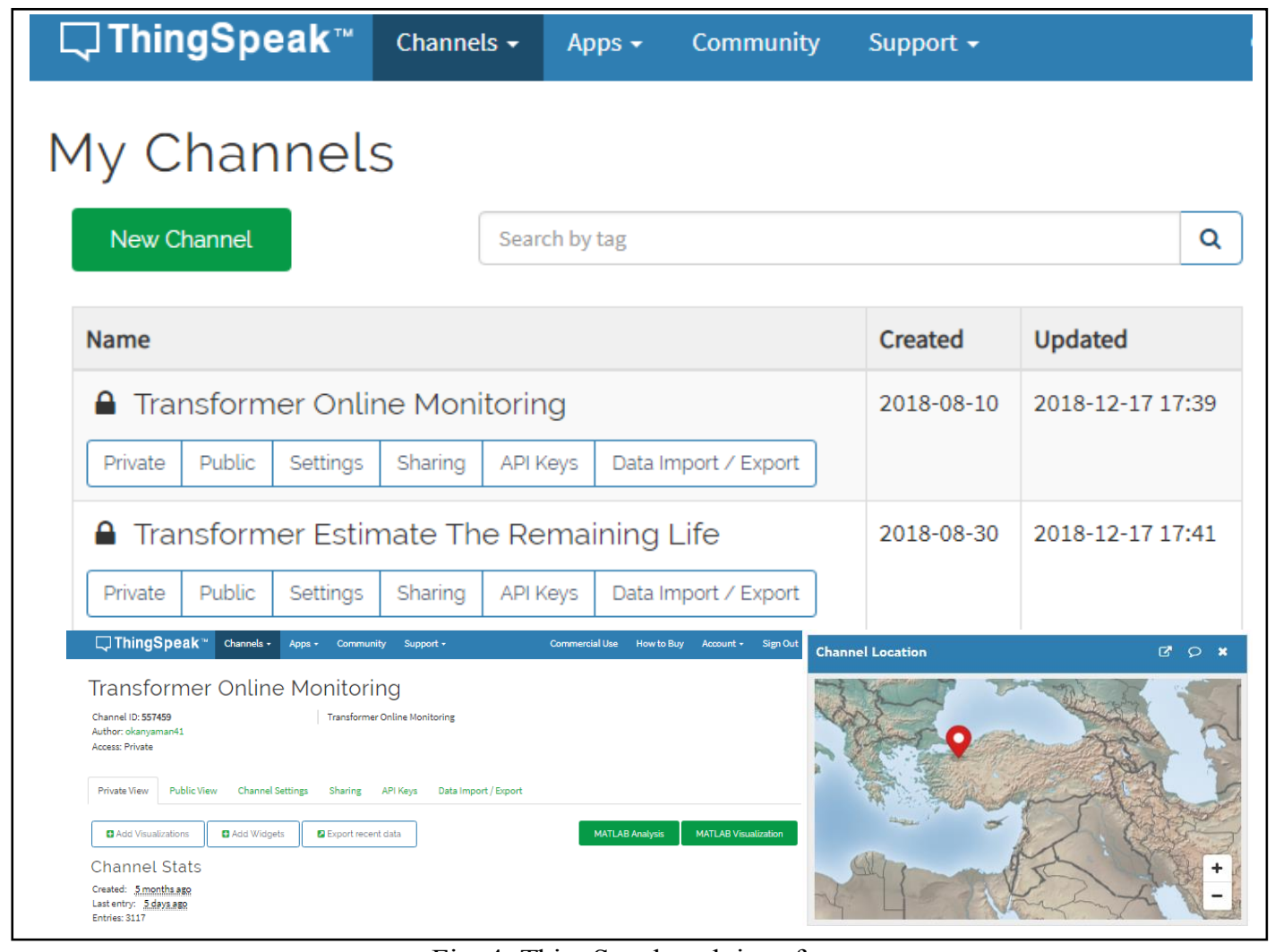

Fig. 4. ThingSpeak web interface 


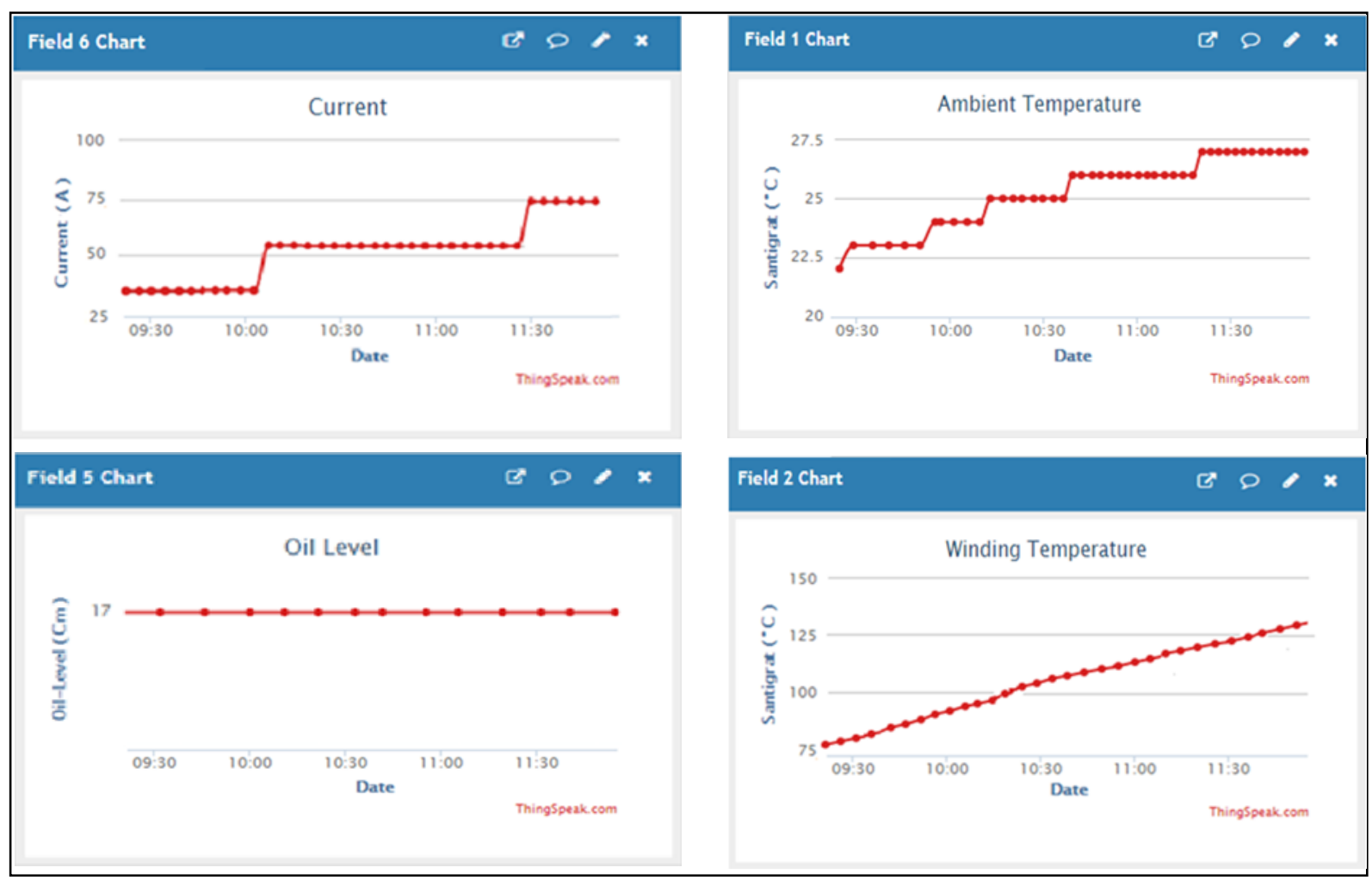

Fig. 5. ThingSpeak sensor data graphical interfaces

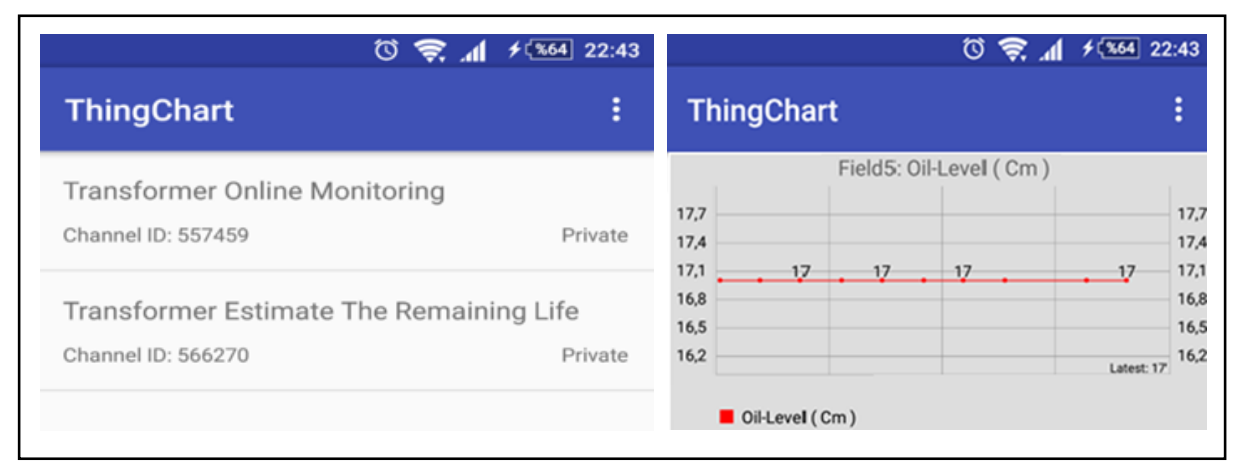

Fig. 6. ThingSpeak mobile interface

\section{PushingBox NotificATION SERVICE}

PushingBox is defined as a cloud that runs with the Internet of Things (IoT) and can send many real time notifications like e-mail, tweet based on API calls [25, 26]. This free platform supports working with the ESP8266 Wi-Fi module. In this way, if any data received from the sensors exceed the critical values, the notification message can be sent to the computer and/or android device with this application. The warning notifications have been tested by deliberately exceeding the limit values, within the scope of this study. For this purpose, an API address has been defined in order to receive notification from PushingBox. Then different notification scenarios have been created with this API address. Fig. 7 show images of PushingBox interfaces.

\section{THE REMAINING SERVICE LIFE ESTIMATION Discussions}

The loss of life calculation and the remaining service life estimation require analytical processing in the monitoring system. According to IEEE-C57.91-2011 standards, the loss of life calculations has been performed with reference to the hotspot temperature in windings [27]. According to the relevant standards, the service life of the transformer in the case of nominal operation is 20.55 years $[28,29]$. However, it is known that the service life of transformers varies with changing load conditions [30,31]. The main reason is that the temperature values in the windings are variable due to the heat loss according to the loading conditions. Since the loading information and the temperature parameters can be received via the monitoring system, it can be performed aging calculations at high resolution as shown in Fig.8. In the meanwhile, the remaining service life of the transformer can also be estimated using the linear approach. 


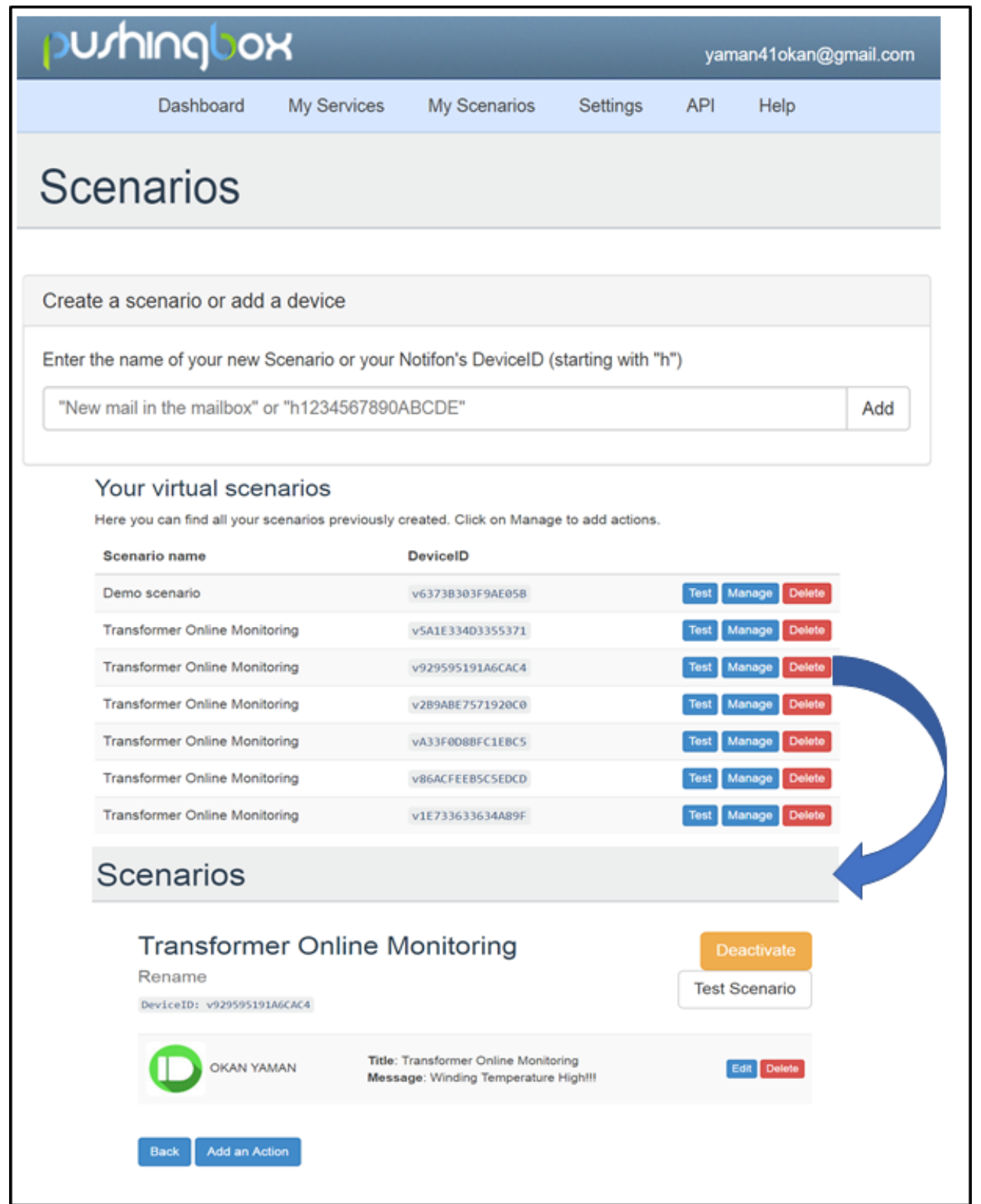

Fig. 7. PushingBox API addresses
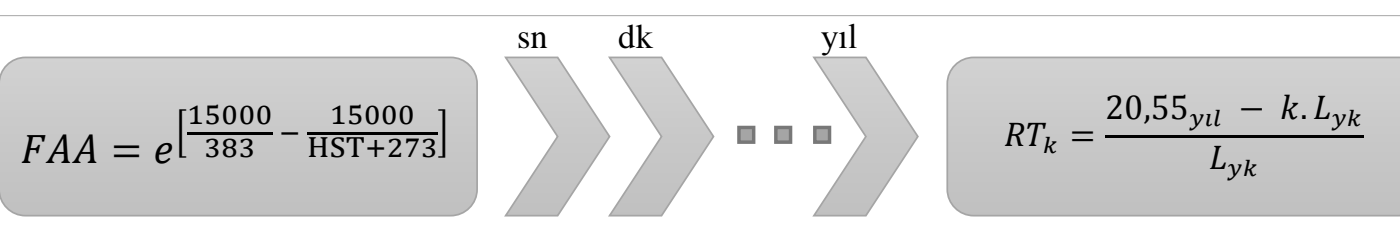

Fig. 8. Remaining service life estimation process

Where, FAA refers to the loss of life at a certain temperature and HST refers to the hot spot temperature. RTk is remaining service life value, $\mathrm{k}$ is year index, Lyk is refers to the total loss of life coefficient up to $\mathrm{k}$ year. The average of the total data obtained from the second period is taken as a single value to the period of minutes. Likewise, the average of the sum of the data obtained from the minute period is shifted to the hourly period. In this way, the loss of life calculated in seconds can be calculated by adding the loss of life in the annual period. In this way, the loss of life calculated in seconds is carried in high resolution up to years. The instant aging acceleration factor, the total loss of life and the remaining service life estimation values can be shown on the LCD display with monitoring system. Similar information can also be displayed as graphical form within the ThingSpeak interface like in Fig. 9. 


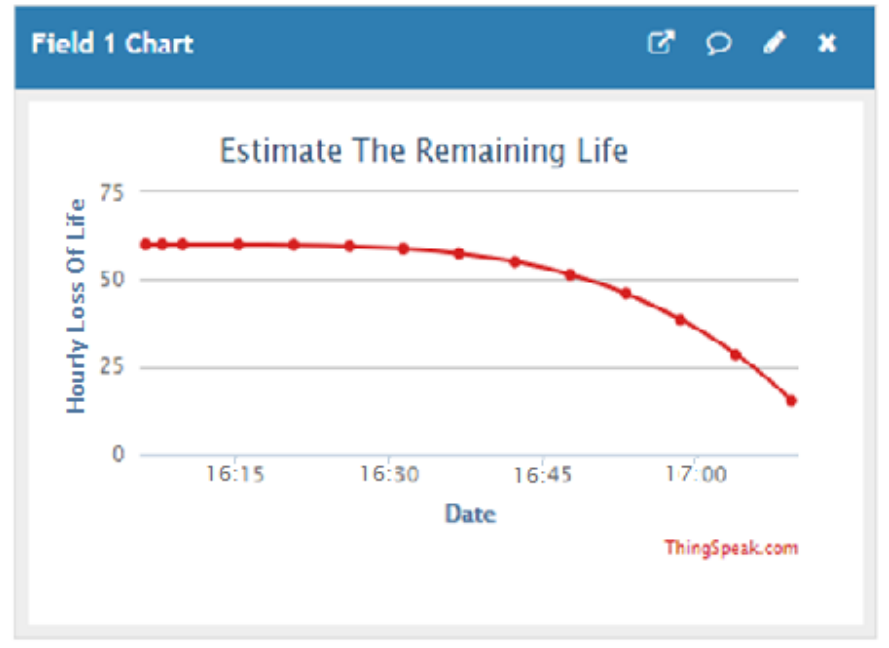

Fig. 9. ThingSpeak service life monitoring

\section{CONCLUSION}

In this study, an original IoT based monitoring system has been developed by using open source software, modular and low cost components. With this system, data can be acquired from different sensors and can be monitored in various environments such as LCD display, computer, mobile phones, and tablets continuously. For this purpose, the ThingSpeak platform free web interface supporting the Internet of Things (IoT) technology has been integrated into the system. In addition, the PushingBox application, which supports IoT technology, can send warning messages without additional hardware and cost. The developed system is capable of performing service life monitoring by using aging equations given in IEEE-C57.91-2011 standards according to change of HST point depending on the loading conditions of transformer. Although the developed system has been designed to monitor a transformer, it is possible to monitor the units in a number of different locations at the same time as replicating similar monitoring platforms. The developed system has been tested on a prototype transformer. It was observed that the data collection, calculation operations and output signals according to the limit values, service life management, recording operations and warning systems were working without error. As a result, this system provides a lowcost alternative to high-cost professional monitoring systems.

\section{REFERENCES}

[1] Su, Yu, et al. "Optimal Planning Method of On-load Capacity Regulating Distribution Transformers in Urban Distribution Networks after Electric Energy Replacement Considering Uncertainties." Energies 11.6 (2018): 1457.

[2] Biçen, Yunus, and Faruk Aras. "Loadability of power transformer under regional climate conditions: the case of Turkey." Electrical Engineering 96.4 (2014): 347-358..

[3] Humayun, Muhammad, et al. "Demand response for operational life extension and efficient capacity utilization of power transformers during contingencies." IEEE Transactions on Power Systems 30.4 (2015): 2160-2169.

[4] Osztermayer, J., et al. "Asset management based on improved online monitoring systems applied to a $110 / 380 \mathrm{kV}$ substation." Power Tech Conference Proceedings, 2003 IEEE Bologna. Vol. 4. IEEE, 2003.
[5] Tenbohlen, Stefan, et al. "Diagnostic measurements for power transformers." Energies 9.5 (2016): 347.

[6] Kun, Wang. "A survey on energy Internet: architecture, approach, and emerging technologies." IEEE Syst J (2017).

[7] Abiri-Jahromi, Amir, et al. "A two-stage framework for power transformer asset maintenance management-Part II: Validation results." IEEE Transactions on Power Systems 28.2 (2013): 1404-1414.

[8] Ma, Hui, et al. "Smart transformer for smart grid—intelligent framework and techniques for power transformer asset management." IEEE Transactions on Smart Grid 6.2 (2015): 1026-1034.

[9] Velasquez-Contreras, Juan L., Miguel A. Sanz-Bobi, and Samuel Galceran Arellano. "General asset management model in the context of an electric utility: application to power transformers." Electric Power Systems Research 81.11 (2011): 2015-2037.

[10] Mariño, Perfecto, et al. "Development of condition monitoring instrumentation for sensing power transformers." INTERNATIONAL CONFERENCE ON SENSING TECHNOLOGY. Vol. 1. 2005.

[11] Islam, Md Mominul, Gareth Lee, and Sujeewa Nilendra Hettiwatte. "A review of condition monitoring techniques and diagnostic tests for lifetime estimation of power transformers." Electrical Engineering 100.2 (2018): 581-605.

[12] Abu-Elanien, Ahmed EB, and M. M. A. Salama. "Asset management techniques for transformers." Electric power systems research 80.4 (2010): 456-464.

[13] Murugan, Raji, and Raju Ramasamy. "Failure analysis of power transformer for effective maintenance planning in electric utilities." Engineering Failure Analysis 55 (2015): 182-192.

[14] Wagle, Anant M., et al. "Real time web based condition monitoring system for power transformers-Case study." Condition Monitoring and Diagnosis, 2008. CMD 2008. International Conference on. IEEE, 2008.

[15] M. Poongothai, P. M. Subramanian, and A. Rajeswari, "Design and implementation of IoT based smart laboratory," 2018 5th Int. Conf. Ind. Eng. Appl. ICIEA 2018, pp. 169-173, 2018.

[16] H. Jamal, M. F. Nadeem Khan, A. Anjum, and M. K. Janjua, "Thermal Monitoring and Protection for Distribution Transformer Under Residential Loading Using Internet of Things," 2018 IEEE Glob. Conf. Internet Things, no. January, pp. 1-6, 2019.

[17] Tenbohlen, S., and F. Figel. "On-line condition monitoring of power transformers." Power Engineering Society Winter Meeting, 2000. IEEE. Vol. 3. IEEE, 2000.

[18] Arabul, Ahmet Yigit, Fatma Keskin Arabul, and Ibrahim Senol. "Experimental thermal investigation of an ONAN distribution transformer by fiber optic sensors." Electric Power Systems Research 155 (2018): 320-330.

[19] Deng, Jian- Gang, et al. "Hot- spot temperature and temperature decay rate measurement in the oil immersed power transformer through FBG based quasi- distributed sensing system." Microwave and Optical Technology Letters 59.2 (2017): 472-475.

[20] Bengtsson, C. "Status and trends in transformer monitoring." IEEE Transactions on Power delivery 11.3 (1996): 1379-1384.

[21] OpenEnergyMonitor, (2019, 6 Mart). [Online]. Erişim: https://learn.openenergymonitor.org/electricity-monitoring/ct-sensors

[22] Bagheri, Mehdi, Amin Zollanvari, and Svyatoslav Nezhivenko. "Transformer Fault Condition Prognosis Using Vibration Signals Over Cloud Environment." IEEE Access 6 (2018): 9862-9874.

[23] Mohamad, Auday AH, Yaqeen S. Mezaal, and Seevan F. Abdulkareem. "Computerized power transformer monitoring based on internet of things." International Journal of Engineering \& Technology 7.4 (2018): 2773-2778.

[24] Meana-Llorián, Daniel, et al. "IoFClime: The fuzzy logic and the Internet of Things to control indoor temperature regarding the outdoor ambient conditions." Future Generation Computer Systems 76 (2017): 275-284.

[25] Fioccola, Giovanni B., et al. "Polluino: An efficient cloud-based management of IoT devices for air quality monitoring." Research and Technologies for Society and Industry Leveraging a better tomorrow (RTSI), 2016 IEEE 2nd International Forum on. IEEE, 2016.

[26] Joshi, Shreedhar A., et al. "Home automation system using wireless network." Communication and Electronics Systems (ICCES), 2017 2nd International Conference on. IEEE, 2017.

[27] IEEE Guide for Loading Mineral-Oil-Immersed Transformers, IEEE C57.91-1995.

[28] Biçen, Yunus, et al. "Aging of Paper Insulation in Natural Ester \& Mineral Oil." Electrical and Electronic Engineering 2.3 (2012): 141-146. 
[29] Agah, SM Mousavi, and H. Askarian Abyaneh. "Quantification of the distribution transformer life extension value of distributed generation." IEEE Transactions on Power Delivery 26.3 (2011): 18201828.

[30] Vaidya, Chinmay V., et al. "Evaluation of High Temperature Operation of Natural Ester Filled Distribution Transformers." 2018 North American Power Symposium (NAPS). IEEE, 2018.

[31] Biçen, Yunus, Faruk Aras, and Hulya Kirkici. "Lifetime estimation and monitoring of power transformer considering annual load factors." IEEE Transactions on Dielectrics and Electrical Insulation 21.3 (2014): 13601367.

\section{BIOGRAPHIES}

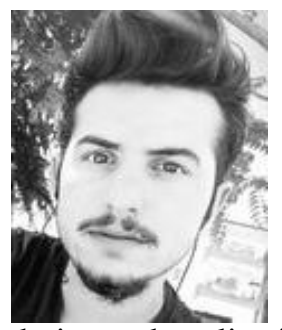

OKAN YAMAN was born in Kocaeli, Turkey in 1995. He received the B.S. Electrical from the University of Tokat Gaziosmanpaşa, Tokat in 2017. He is currently an MSc student in Institute of Science and Technology at the University of Duzce. His research interests include automation systems, electronic circuit design and applications.

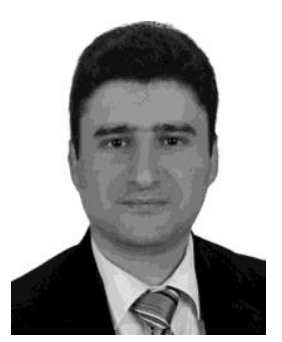

YUNUS BICEN was born in Kastamonu, Turkey in 1981. He received the BSc degree from Kocaeli University, Electrical Education Department, in 2004, the MSc and $\mathrm{PhD}$ degrees from Kocaeli University, Turkey in 2007 and 2012 respectively. He was with the University of Kocaeli between 2004 and 2008. Currently, he works as an Associate Professor at the Department of Electrical-Electronics Engineering - Duzce University. His research interests are in the area of power systems analysis, aging of power system equipment, fault diagnostics and development of computer aided programs. 